Communications in Physics, Vol.26, No. 1 (2016), pp. 59-66

DOI:10.15625/0868-3166/26/1/7686

\title{
MAGNETIC FIELD ASSISTED MELT-SPINNING Nd-Fe-B RIBBONS AND ANISOTROPIC BONDED NdFeB MAGNETS PREPARED THEREOF
}

\author{
NGUYEN XUAN TRUONG AND NGUYEN VAN VUONG ${ }^{\dagger}$ \\ Institute of Materials Science, Vietnam Academy of Science and Technology, \\ 18 Hoang Quoc Viet Street, Cau Giay District, Hanoi, Vietnam \\ ${ }^{\dagger} E$-mail: vuongnv@ims.vast.ac.vn \\ Received 08 January 2016 \\ Accepted for publication 14 April 2016
}

\begin{abstract}
The magnetic-field-assisted melt-spinning (FAMS) $\mathrm{Nd}_{10.5} \mathrm{Fe}_{72} \mathrm{Co}_{11} B_{6.5}$ ribbons were melt-spun in an external magnetic field of $3.2 \mathrm{kG}$. The prepared ribbons were ball-milled in xylene solvent for 30 minutes followed by adding in and mixing with the high-temperature binder HTB-1 (3wt\%) for further 10 minutes. The mixed powder was dried and in-mold aligned in a magnetic field intensity of $18 \mathrm{kOe}$ and hot-compacted at $200^{\circ} \mathrm{C}$. The parameters of the FAMS ribbon preparation, ball-milling process, hot-compaction and anisotropic bonded magnets' fabrication were optimized leading to the magnet's energy product $(\mathrm{BH})_{\max }$ of $10 \mathrm{MGOe}$. The microstructure and magnetic properties of the prepared ribbons and bonded magnets will be discussed in details.
\end{abstract}

Keywords: field-assisted melt-spinning (FAMS), magnetic properties, anisotropic magnets.

Classification numbers: 61.05.cp, 75.30.Gw, 75.50.Ww, 75.60.Ej.

\section{INTRODUCTION}

By the conventional melt-spinning technique, the $\mathrm{Nd}_{2} \mathrm{Fe}_{14} \mathrm{~B}$ ribbons are formed with the microstructure where the grains are randomly oriented. These ribbons are utilized for making isotropic bonded magnets by using an organic binder. Because of the random orientation of easy axes, the remanent magnetization $M_{r}$ of ribbons is $\sim 0.5 M_{s}=8 \mathrm{kG}$. It is understood that the microstructure of aligned grains is desired to enhance the remanence which, in turn, can improve the squareness and the energy product $(B H)_{\max }$ of ribbons and magnets prepared thereof, if the coercivity of ribbons and magnets should be well controlled.

Several routes have been explored with the aim of producing anisotropic $\mathrm{NdFeB}$ hard nanostructured materials by HDDR treatments [1-5]. Different techniques were applied to prepare anisotropic bulk magnets like the die-upset or hot deformation methods [6-9]. However, these methods are difficult to be employed for massive production of magnets.

(C)2016 Vietnam Academy of Science and Technology 
Recently, the nanocomposite hard/soft magnets are developed from bottom-up. The nanocomposite core/shell grains are the building blocks of this kind of magnets. Normally, the configuration of hard core/soft shell is used for the nanostructured nanocomposite hard/soft magnet development. We proved in Ref. [10] that this configuration can be easily prepared by the magnetic-field-assisted melt-spinning (FAMS) technique. For Nd-Fe-B systems, during the FAMS process, the $\alpha$-Fe seeds are formed initially on the wheel surface, the hard magnetic Nd-Fe-B grains are then grown on the seeds along the temperature gradient perpendicular to the ribbon plane. This crystalline growth process creates (001) texture of ribbons. The magnetic field increases the volume energy of seeds and thus decreases their critical size and, consequently, decreases the average grain size [10-12]. These textured ribbons should become a good starting material for preparing anisotropic magnets.

In this work, we present the results of preparing the anisotropic nanocomposite $\mathrm{Nd}_{2}(\mathrm{Fe}, \mathrm{Co})_{14} \mathrm{~B} / \alpha$-Fe ribbons by using the FAMS process. The FAMS ribbons consist of nanocomposite hard core/soft shell grains and were used for producing anisotropic bonded magnets.

\section{MATERIALS AND METHODS}

The pre-alloy of composition of $\mathrm{Nd}_{10.5} \mathrm{Fe}_{72} \mathrm{Co}_{11} \mathrm{~B}_{6.5}$ was arc-melted under argon protection atmosphere by using the starting high-purity (99.9\%) metals of $\mathrm{Nd}, \mathrm{Fe}, \mathrm{Co}$ and $\mathrm{FeB}$ alloy. The ingot was melted thrice to obtain a high homogeneity. In a typical procedure, the batch amount of $\sim 20 \mathrm{~g}$ of this pre-alloy was melt-spun onto a copper wheel under $0.05 \mathrm{MPa}$ argon atmosphere from a quartz tube. The copper wheel has been equipped with the magnetic field oriented perpendicular to the wheel surface. The field strength measured directly on the wheel surface was $3.2 \mathrm{kG}$. The quartz tube orifice diameter was fixed at $1.0 \mathrm{~mm}$, the distance between the nozzle and the wheel surface was kept constant by $5 \mathrm{~mm}$. The copper wheel speed was varied in the range of $8-16$ $\mathrm{m} / \mathrm{s}$, which was lower than that chosen in [10]. The composition and structure were examined by the X-ray diffraction (XRD) patterns carried out by using SIEMENS D5000 X-ray diffractometer with $\mathrm{Cu}-\mathrm{K}_{\alpha}$ radiation. The phase composition was analyzed by means of Rietveld refinement of XRD patterns for all the diffraction peaks taken in the $2 \theta$ angle ranged from $25^{\circ}$ to $85^{\circ}$ by using the Crystal Impact Software for Chemists and Material Scientists. The texture of magnets was determined by using the alignment degree of the (001) peaks appeared on the XRD patterns taken on the magnet surface [13]. The morphology of ribbons was studied by using the HitachiS4800 field emission scanning electron microscopy (FESEM). The hysteresis loops of ribbons were measured by the pulsed field magnetometer (PFM) under an applied field up to $60 \mathrm{kOe}$.

\section{RESULTS AND DISCUSSION}

\section{The field-assisted melt-spinning ribbons}

The wheel speeds ranged from 8 to $16 \mathrm{~m} / \mathrm{s}$ are found to be optimal to form hard/soft core/shell grains aligned parallel to the direction of the assisted magnetic field. This range of wheel speeds optimizes two requests: the growth of grains with suitable sizes to maintain a high coercivity and the alignment of easy axes of grains along the direction of the magnetic field assisting the melt-spinning process.

The XRD patterns of $\mathrm{Nd}_{10.5} \mathrm{Fe}_{72} \mathrm{Co}_{11} \mathrm{~B}_{6.5}$ ribbons are shown in Fig. 1. The magnetic texture of the free surface of ribbons were clearly observed by the significant increase of intensities of 
(004), (006), (008) and $(00,10)$ peaks. This crystallographic preferred orientation along c-axis was caused by the shape anisotropy of Fe/Co seeds formed at the beginning step of crystal growth under action of an assisting field [13]. The texture was enhanced in the ribbon melt-spun at the lowest used speed $\mathrm{v}=8 \mathrm{~m} / \mathrm{s}$ and is due to the synchronal effect of two forces creating the texture, the field assisted $[10,11,14]$ and the temperature gradient oriented perpendicularly to the ribbon plane. This gradient was established by the rapid cooling of the melt dropped on the rotating wheel surface [14]. The formed texture helped to enhance the squareness $\gamma$ of the ribbon magnetization loops which can be, for the $\mathrm{Nd}-\mathrm{Fe}-\mathrm{B}$ based ribbons with high crystalline anisotropy, evaluated by using the ratio between two coercivities, $\gamma={ }_{b} \mathrm{H}_{c} /{ }_{i} \mathrm{H}_{c}$. The values of $\gamma$ are $0.81,0.75,0.73,0.70$ and 0.71 for the FAMS ribbons melt-spun in $3.2 \mathrm{kG}$ at $\mathrm{v}=8,10,12,14$ and $16 \mathrm{~m} / \mathrm{s}$, respectively.

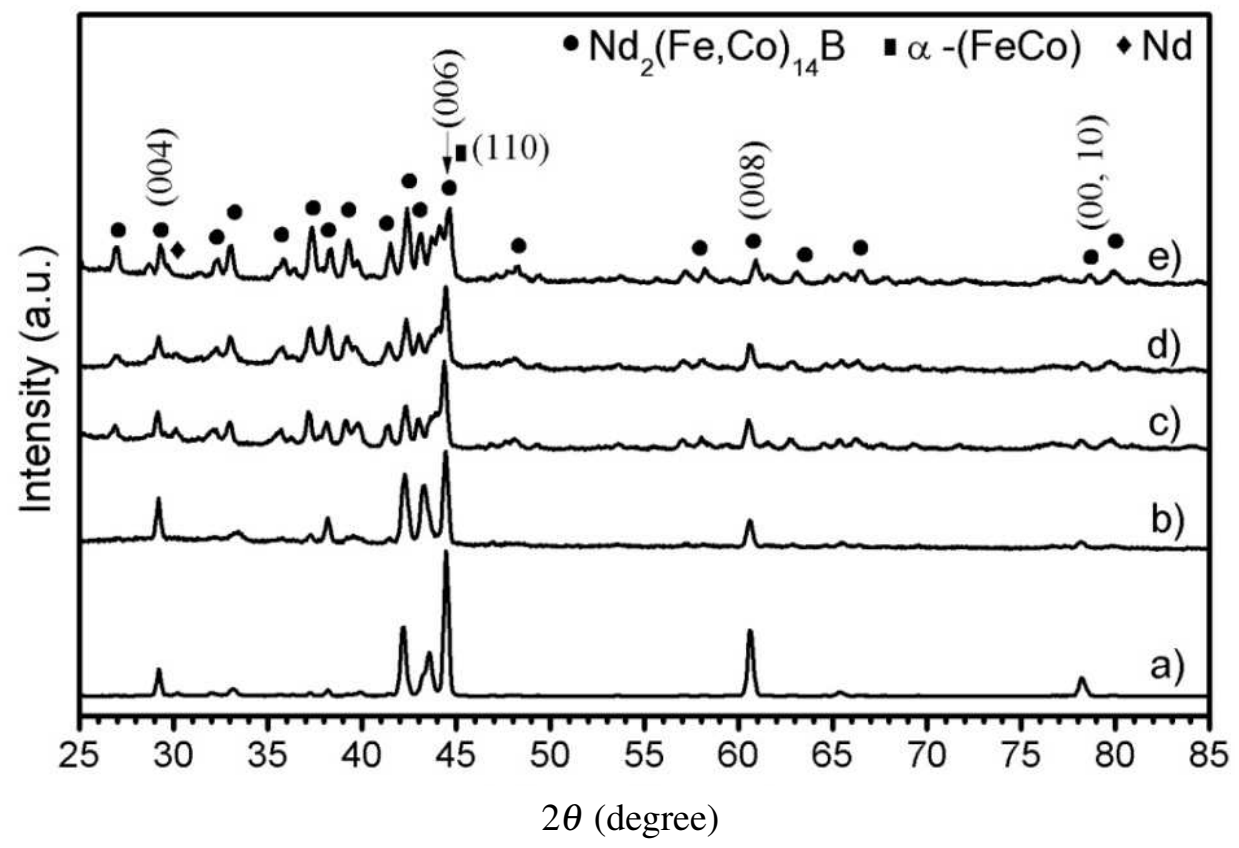

Fig. 1. XRD patterns of FAMS $\mathrm{Nd}_{10.5} \mathrm{Fe}_{72} \mathrm{Co}_{11} \mathrm{~B}_{6.5}$ ribbons melt-spun at: a) $v=8 \mathrm{~m} / \mathrm{s}$, b) $v=10 \mathrm{~m} / \mathrm{s}$, c) $v=12 \mathrm{~m} / \mathrm{s}, \mathrm{d}) v=14 \mathrm{~m} / \mathrm{s}$; e) $v=16 \mathrm{~m} / \mathrm{s}$.

The mentioned texture of the prepared FAMS Nd ${ }_{10.5} \mathrm{Fe}_{72} \mathrm{Co}_{11} \mathrm{~B}_{6.5}$ ribbons is approved by the high ratio $\alpha$ between the remanent magnetization $M_{r}\{M(H=0)\}$ and the magnetization $M_{s}$ measured at $H=30 \mathrm{kOe}$ (see Fig. 2). For the ribbons melt-spun at $\mathrm{v}=8,10,12,14$ and $16 \mathrm{~m} / \mathrm{s}$, the factor $\alpha$ is $0.66,0.69,0.73,0.68$ and 0.70 , respectively. All these values are higher than the value of 0.5 typical for the isotropic ribbons.

To analyze the phase composition of melt-spun ribbons the Rietveld refinement method was used. The typical result is presented on Fig. 3. The refinement of the XRD pattern of ground $\mathrm{Nd}_{10.5} \mathrm{Fe}_{72} \mathrm{Co}_{11} \mathrm{~B}_{6.5}$ ribbon melt-spun in $3.2 \mathrm{kG}$ and at $v=12 \mathrm{~m} / \mathrm{s}$ reveals the coexistence of the hard phase of $\mathrm{Nd}_{2} \mathrm{Fe}_{12.8} \mathrm{Co}_{1.2} \mathrm{~B}$ (phase 96-151-1058), the soft phase Fe/Co and the non-magnetic phase of $\mathrm{Nd}$. The average grain size is about $100 \mathrm{~nm}$ which coincides with the SEM result attached in the inset of Fig. 3. 


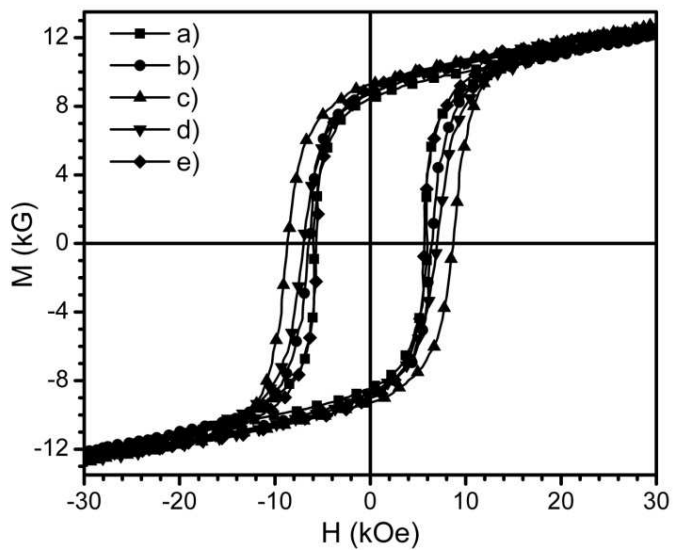

Fig. 2. $\mathrm{M}(\mathrm{H})$ curves of the FASM ribbons melt-spun at: a) $v=8 \mathrm{~m} / \mathrm{s}, \mathrm{b}) v=10 \mathrm{~m} / \mathrm{s}, \mathrm{c})$ $v=12 \mathrm{~m} / \mathrm{s}, \mathrm{d}) v=14 \mathrm{~m} / \mathrm{s}, \mathrm{e}) v=16 \mathrm{~m} / \mathrm{s}$.

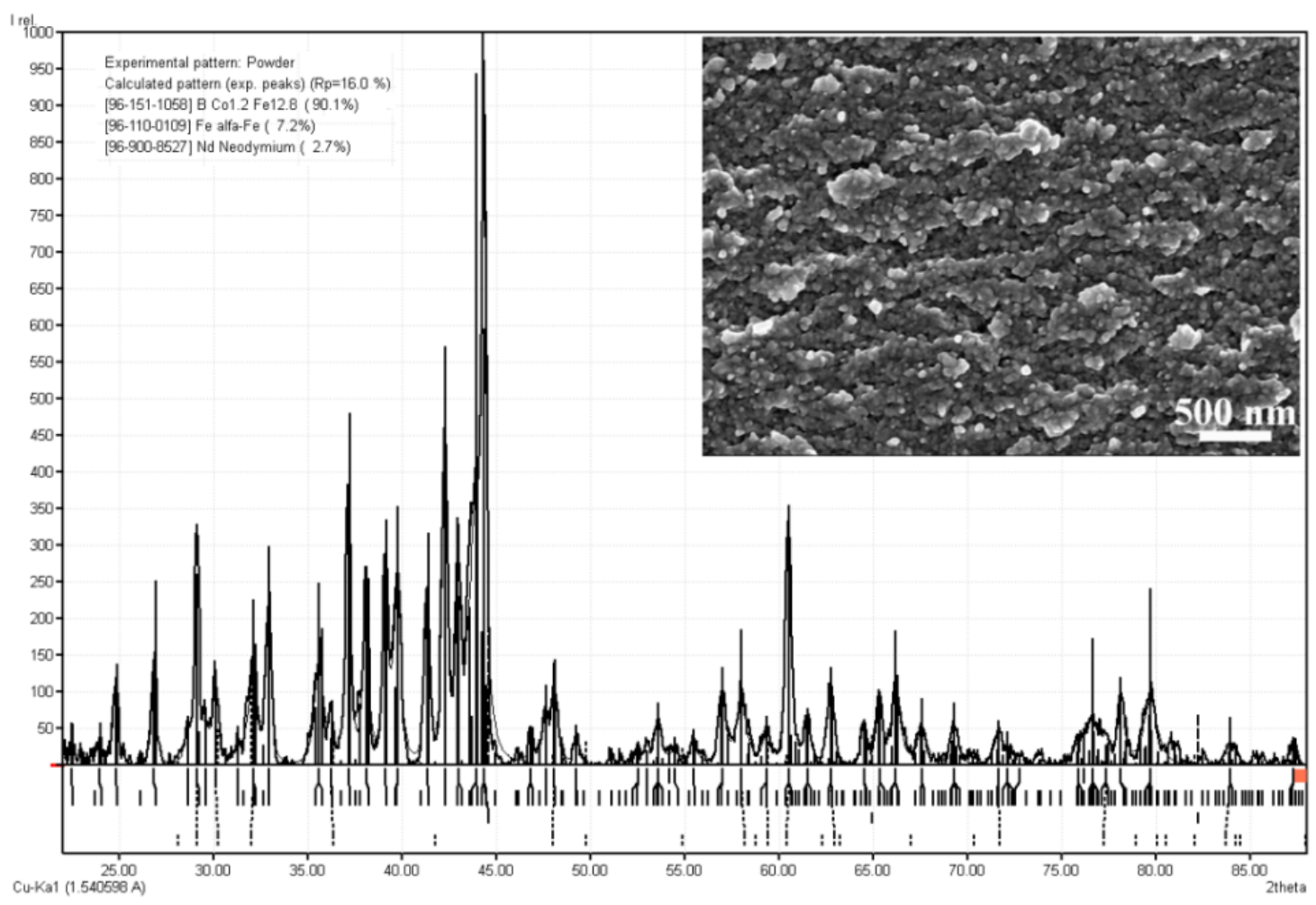

Fig. 3. The Rietveld refinement result of the FASM $\mathrm{Nd}_{10.5} \mathrm{Fe}_{72} \mathrm{Co}_{11} \mathrm{~B}_{6.5}$ ribbon meltspun in $3.2 \mathrm{kG}$ and at $v=12 \mathrm{~m} / \mathrm{s}$. Its FESEM graph is attached in the inset.

The soft phase fraction was estimated by the Rietveld refinement is about $7 \mathrm{wt} \%$. The existence of this soft phase is approved by the non-zero magnetization of the temperature dependent magnetization curve $M(T)$ measured for the range $T>673 \mathrm{~K}$ (see Fig. 4). The abrupt 
decrease of $M(T)$ curve corresponds to the Curie temperature $T_{c}$, where the ferromagnetic to paramagnetic phase transition is occurred. The enhancement of $T_{c}$ in comparison with the value of $593 \mathrm{~K}$ of $\mathrm{Nd}_{2} \mathrm{Fe}_{14} \mathrm{~B}$ phase is resulted by the partial substitution of Fe by Co in this $7 \mathrm{wt} \%$ of the soft phase [15].

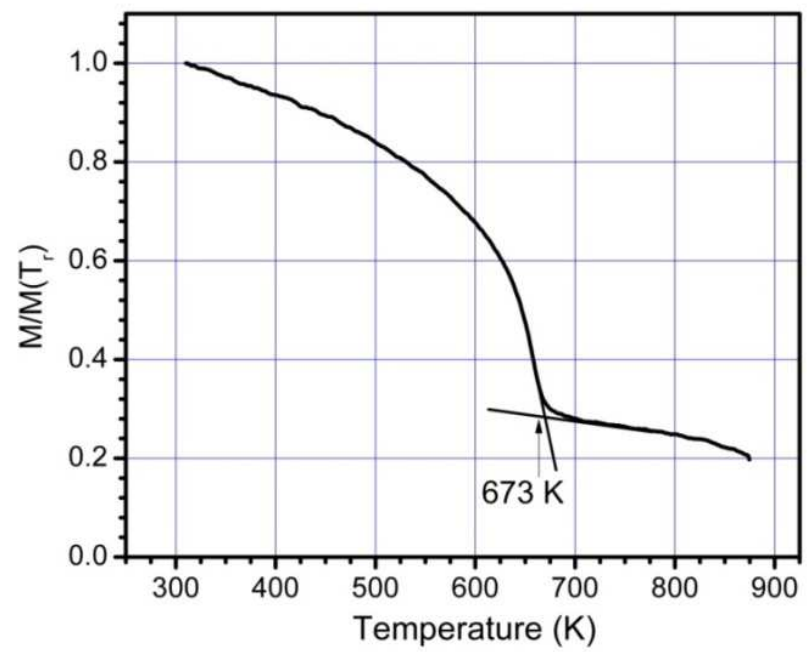

Fig. 4. Thermo-magnetic curve $\mathrm{M}(\mathrm{T})$ of the $\mathrm{Nd}_{10.5} \mathrm{Fe}_{72} \mathrm{Co}_{11} \mathrm{~B}_{6.5}$ ribbon melt-spun at $v=$ $12 \mathrm{~m} / \mathrm{s}$. The field of $0.5 \mathrm{kOe}$ was applied during this measurement. The sample was thermally demagnetized before the measurement.

\section{Preparation of anisotropic magnets}

The highly coercive ribbons melt-spun in $3.2 \mathrm{kG}$ at the wheel speed of $12 \mathrm{~m} / \mathrm{s}$ was chosen for making anisotropic bonded magnets. The one-component high-melting temperature binder was added to the ribbons in the weighing ratio of binder to ribbons of 3:100. The mixture was ball-milled into the powders with the average size of $\sim 5 \mu \mathrm{m}$. The final powders were loaded into the mold, aligned in $18 \mathrm{kOe}$ magnetic field and hot-compacted at $200^{\circ} \mathrm{C}$ under 4000 psi for 30 minutes.

The prepared magnet has the mass density of $\sim 6.0 \mathrm{~g} / \mathrm{cm}^{3}$. The anisotropy of magnets was checked by analyzing the XRD patterns (see Fig. 5A) taken on the magnet surface with the normal vector perpendicular (perpendicular surface, curve a) and parallel (parallel, curve b) to the direction of the aligning magnetic field.

The intensity enhancement of two peaks (004), (006) of the pattern a) of Fig. 5A) reveals the texture enhancement of the parallel surface in comparison with that of the perpendicular one. The ratio $\zeta$ between the two peaks, $\zeta=I_{(006)} / I_{(410)}$, was taken into account for estimating the texture degree of crystalline alignment along the easy axis (001). For the fully isotropic $\mathrm{Nd}_{2} \mathrm{Fe}_{12.8} \mathrm{Co}_{1.2} \mathrm{~B}$ powder sample (see Fig. 5B), $\zeta$ equals 0.34 which is 5.3 times lower than the value of $\zeta=1.81$ 
calculated from the pattern $\mathrm{b}$ of Fig. 5A for the parallel surface, which, in turn, is about 2 times larger than that $\zeta$ calculated from the curve a of Fig. 5A for the perpendicular surface.
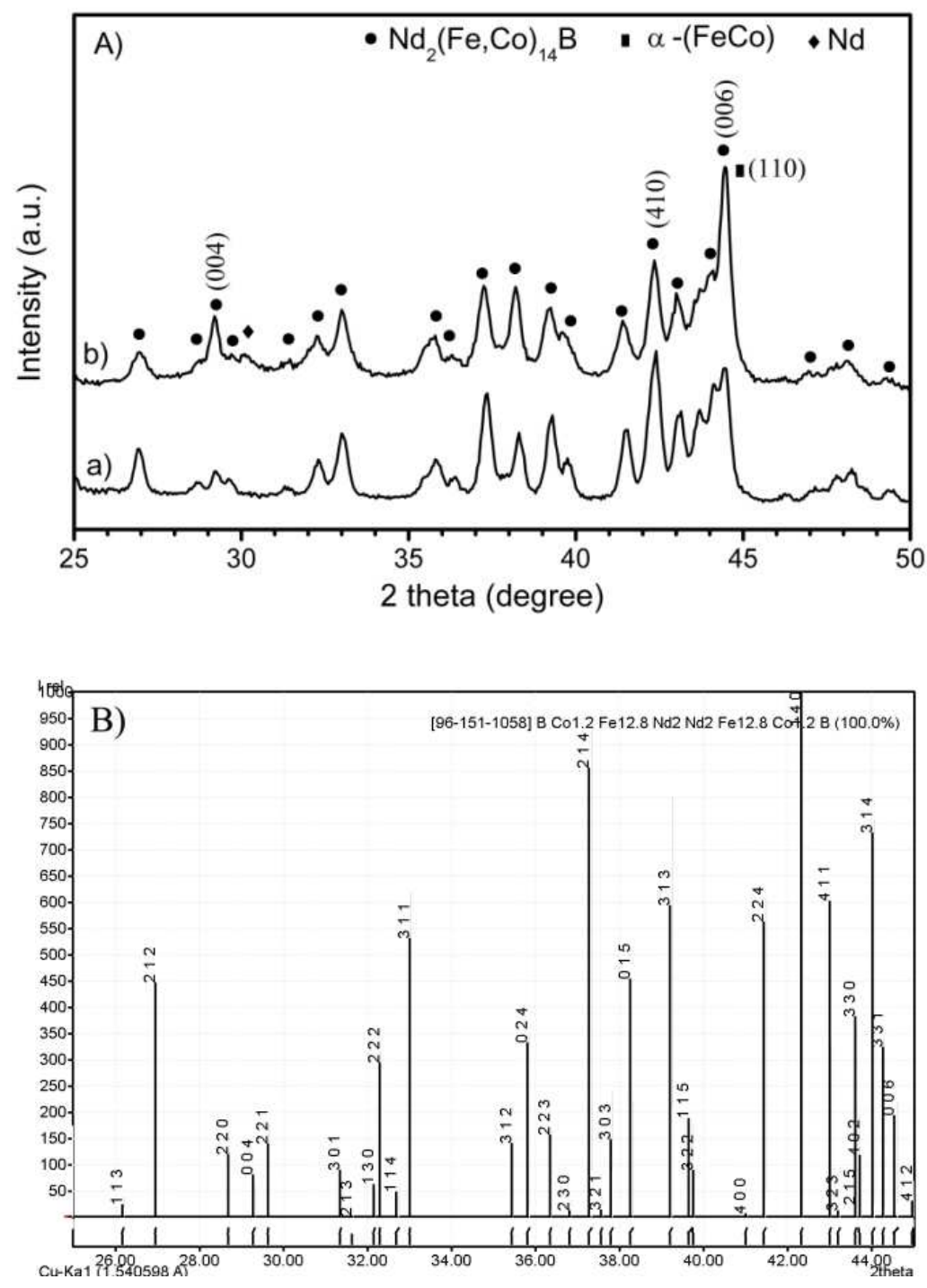

Fig. 5. A) XRD pattern of the magnet taken on the: a) perpendicular surface, b) parallel surface; B) Standard calculated XRD pattern for the fully isotropic powder of $\mathrm{Nd}_{2} \mathrm{Fe}_{12.8} \mathrm{Co}_{1.2} \mathrm{~B}$ (phase 96-151-1058).

The magnet's anisotropy observed in Fig. 5A is supported by the discrepancy of the magnet's demagnetization curves measured with the measuring field oriented parallel and perpendicular to the alignment direction. These two curves are presented in Fig. 6. For these measurements, 
the samples were cut from the magnet with the aspect ratio larger than 5 to avoid the demagnetization effect.

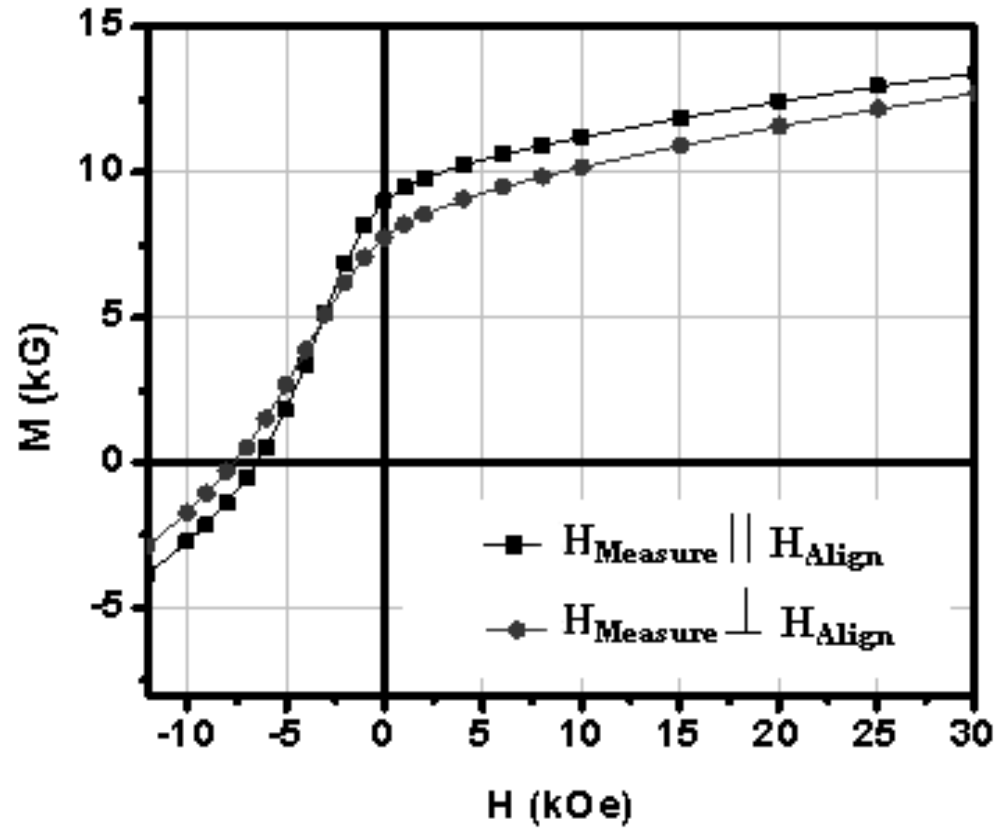

Fig. 6. The magnetization loops of the $\mathrm{Nd}_{10.5} \mathrm{Fe}_{72} \mathrm{Co}_{11} \mathrm{~B}_{6.5}$ magnet measured in the field oriented perpendicular $(\bullet)$ and parallel $(\boldsymbol{\square})$ to the alignment direction.

The remanent magnetization $M_{r}$ of the parallel sample is $8.8 \mathrm{kG}$ which is in $1.2 \mathrm{kG}$ larger than that of the perpendicular sample. The quantitative alignment degree of the magnet can be described by the average misalignment angle, $\psi=\arctan \left[2 M_{r \perp} / M_{r \|}\right]$, where $M_{r \perp}$ and $M_{r \|}$ are the remanences measured for the perpendicular and parallel samples, respectively [16]. Typically, $\psi=0$ for the perfect alignment and $90^{\circ}$ for the non-alignment. In our case, $\psi$ equals $\sim 56^{\circ}$, corresponding to the moderate alignment. This alignment degree allows the intrinsic coercivity ${ }_{i} H_{C}=7.0 \mathrm{kOe}$ and $(B H)_{\max }=10 \mathrm{MGOe}$.

\section{CONCLUSIONS}

High-performance Field-Assisted Melt-Spining (FAMS) $\mathrm{Nd}_{10.5} \mathrm{Fe}_{72} \mathrm{Co}_{11} \mathrm{~B}_{6.5}$ ribbons were melt-spun in $3 \mathrm{kG}$ at the optimal wheel speed of $12 \mathrm{~m} / \mathrm{s}$. The nanocomposite microstructure of ribbons was proved by the XRD, FESEM, $M(H)$ and $M(T)$ measurements. These high-performance ribbons were used to produce anisotropic bonded magnets. The good alignment degree of prepared magnets was revealed by the XRD patterns measured on the perpendicular and parallel surfaces of magnets. The moderate misalignment angle $\psi \sim 56^{\circ}$ was determined by using the remanences measured for the perpendicular and parallel samples. The best value of the energy product $(B H)_{\max }$ of the prepared magnets is $10 \mathrm{MGOe}$, which meets the technical standards required for bonded magnet applications. The investigation is being carried out for further enhancement of the performance of anisotropic magnets. 


\section{ACKNOWLEDGEMENTS}

This research is funded by the Key National Laboratory of the Institute of Materials Science, under Grand CST02.14. The authors also gratefully thank Holger Putz, who created match version 3.0.

\section{REFERENCES}

[1] Choi P., Tomida T., Maehara Y., Uehara M., and Hirosawa S., Proceedings of the 2nd Pacific Rim Conference on Advanced Materials and Processing (1995) 1401-1406.

[2] Gutfleisch O., Gebel B., and Mattern N., J. Magn. Magn. Mater. 201 (2000) L5-L9.

[3] Liu M., Xiao R., Gao R.W., and Han G.B., Physica B 406 (2011) 3534-3537.

[4] Rybalka S.B., Goltsov V.A., Didus V.A., and Fruchart D., J. Allloys. Comp. 356-357 (2003) 390-394.

[5] Tomida T., Choi P., Maehara Y., Uehara M., Tomizawa H., and Hirosawa S., J. Allloys. Comp. 242 (1996) 129-135.

[6] D. C. Crew, L. H. Lewis, and V. Panchanathan, J. Magn. Magn. Mater. 223 (2001) 261-266.

[7] R. W. Lee, Appl. Phys. Lett. 46 (1985) 790-791.

[8] S.Guruswamy, Y.R.Wang, and V.Panchanathan, J. Appl. Phys. 83 (1998) 6393-6395.

[9] Y. R. Wang, S. Guruswamy, and V. Panchanathan, J. Appl. Phys. 81 (1997) 4450-4452.

[10] Nguyen Xuan Truong, Vu Hong Ky, Do Hung Manh, Nguyen Van Khanh, and Nguyen Van Vuong, Advances in Materials Science and Engineering 2013 (2013) 927356, doi:http://dx.doi.org/10.1155/2013/927356.

[11] Nguyen Xuan Truong, Nguyen Trung Hieu, Vu Hong Ky, and Nguyen Van Vuong, Journal of Nanomaterials, 2012 (2012) 759750, doi:http://dx.doi.org/10.1155/2012/759750.

[12] Nguyen Xuan Truong, Vu Hong Ky, Nguyen Van Khanh, and Nguyen Van Vuong, Proceedings of the 6th International Workshop on Advanced Materials Science and Nanotechnology (2012) 253-257.

[13] Vuong Van Nguyen, Chuanbing Rong, Yong Ding, and J. Ping Liu, J. Appl. Phys. 111 (2012) 07A731.

[14] X. Y. Zhang, Y. Guan, L. Yang, and J. W. Zhang, Appl. Phys. Lett. 79 (2001) 2426.

[15] Z. W. Liu, and H. A. Davies, Journal of Physics D: Applied Physics 39 (2006) 2647.

[16] W. Fernengel, A. Lehnert, M. Katter, W. Rodewald, and B. Wall, Journal of Magnetism and Magnetic Materials 157-158 (1996) 19-20. 